\title{
Using Open-Source Components to Process Interferometric TerraSAR-X Spotlight Data
}

\author{
Michael Jendryke, ${ }^{1,2}$ Timo Balz, ${ }^{1}$ Houjun Jiang, ${ }^{1}$ Mingsheng Liao, ${ }^{1}$ and Uwe Stilla ${ }^{2}$ \\ ${ }^{1}$ State Key Laboratory of Information Engineering in Surveying, Mapping and Remote Sensing, Wuhan University, \\ Wuhan, Hubei 430079, China \\ ${ }^{2}$ Photogrammetry and Remote Sensing, Technische Universitaet Muenchen, 80333 Munich, Germany \\ Correspondence should be addressed to Michael Jendryke; michael.jendryke@rub.de
}

Received 21 November 2012; Accepted 20 January 2013

Academic Editor: Gui Gao

Copyright (c) 2013 Michael Jendryke et al. This is an open access article distributed under the Creative Commons Attribution License, which permits unrestricted use, distribution, and reproduction in any medium, provided the original work is properly cited.

We address the processing of interferometric TerraSAR-X and TanDEM-X spotlight data. Processing steps necessary to derive interferograms at high spatial resolution from bi- and monostatic satellite images will be explained. The spotlight image mode is a beam steering technique focusing the antenna on a specific ground area. This results in a linear Doppler shift frequency in azimuth direction, which has to be matched to the master image. While shifting the interpolation kernel in azimuth during resampling, the frequency spectrum of the slave image is aligned to the master image. We show how to process bistatic TanDEM-X images and propose an integrated processing option for monostatic TerraSAR-X data in the Delft Object-oriented Radar Interferometric Software (DORIS). The paper focuses on the implementation of this algorithm for high-resolution spotlight InSAR in a public domain tool; hence, it becomes available to a larger research community. The results are presented for three test areas: Uluru in Australia, Las Vegas in the USA, and Lüneburg in Germany.

\section{Introduction}

Radar remote sensing with SAR (Synthetic Aperture Radar) satellites is used to observe the Earth from space $(\sim 500 \mathrm{~km}$ away) [1]. It is a space-borne image acquisition technique that is able to penetrate cloud coverage and that is not dependent on sunlight. The Earth's surface is illuminated with electromagnetic waves sent by the satellite antenna $[2,3]$. The backscattered signal is received by the same antenna (monostatic) or other antennas (bi- or multistatic) and is stored as a complex image with amplitude and phase information [4]. SAR images have a broad range of applications such as urban surveillance [5, 6], damage assessment [7-9], glacier monitoring [10], and deformation mapping [11, 12].

New generation satellites as TerraSAR-X or COSMOSkyMed provide images in single polarization with a resolution of approximately $1 \mathrm{~m}$ (with $300 \mathrm{MHz}$ bandwidth) [13]. The highest-resolution image mode is called spotlight mode and covers-compared to other image modes-a relatively small ground area [13]. TerraSAR-X and COSMO-SkyMed operate in the X-band spectrum, which is particularly useful for observing man-made structures. High resolution is of interest when monitoring small parts of urban areas and single building structures [5]. In order to achieve resolution in the meter or submeter region, the antenna beam is steered backwards, while the satellite passes the area of observation [14]. One limitation is that continuous acquisition along the ground track is not possible. A full spotlight scene with two subsets is shown in Figure 1.

A consequence of beam steering is the linear Doppler shift, that causes missmatched image spectra, which have to be aligned in order to create spotlight interferograms [14]. The ground area has to be sampled more often to achieve high spatial resolution, which is only possible by increasing the illumination time. Therefore, the beam is directed forward (from the perspective of the satellite) and gradually motioned backwards as the antenna passes the area of interest [13]. This means a positive Doppler frequency at the first image line, which is linearly declining line by line, reaching a single line with zero Doppler in the image center and negative 


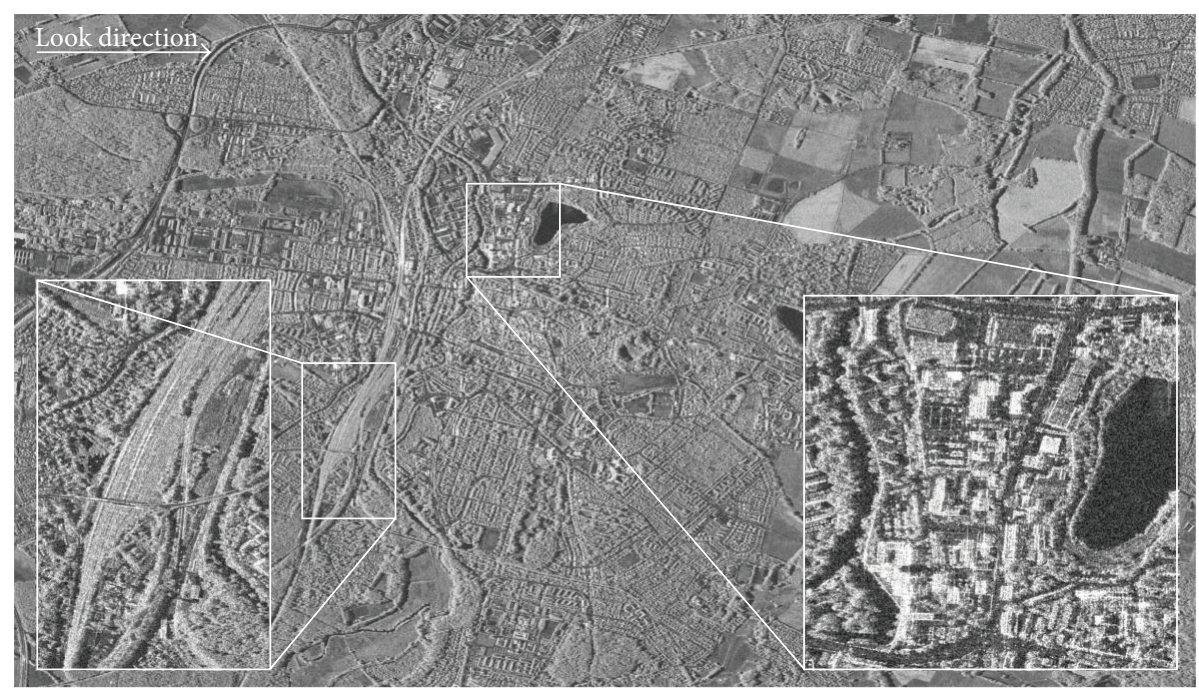

FIGURE 1: TerraSAR-X Spotlight image (full scene) of Lüneburg, Germany. Left subset shows a railway station, right subset shows building structures and a pond, (C) DLR 2010.

frequencies towards the last line. Matching the two image spectra is the challenge in interferometric processing of spotlight data.

The purpose of this paper is (i) to explain high-resolution spotlight interferogram generation for bi- and monostatic acquisitions, (ii) the implementation of a resampling algorithm described in detail in Eineder et al. [14], and (iii) making it available to the research community by incorporating the source code into DORIS [15]. This will enable researchers to generate coherent high-resolution interferograms with an open software solution. The key point of this paper lies in the instruction to process bi- and monostatic acquisitions.

In this paper, we show TanDEM-X single pass interferometry (bistatic) and TerraSAR-X (TSX) repeat pass interferometry (monostatic). We abbreviate the TerraSAR-X satellite as TSX, TanDEM-X as TDX, and the bistatic product, generated by the satellite constellation, as TDM. In the first (bistatic) case, one satellite serves as a sender to illuminate the ground. Both antennas on satellite 1 (TSX) and 2 (TDX) are used for receiving [16]. In order to form images in this mode, the spacecrafts have to orbit in close formation and must be linked together to acquire two images at almost the same time from slightly different positions [4]. In the second case, one satellite serves as a sending and receiving antenna during one image acquisition. Images are recorded every time the satellite passes the area of interest.

This article is divided into four parts starting from simple to more complex operations. It will first discuss TDM spotlight products (TanDEM-X bistatic) and the additional processing steps required to generate a bistatic interferogram. The second part illustrates how proper spotlight interferograms are derived by describing how spotlight images are acquired and subsequently treated during the resampling process. The last two parts show examples and discuss the results, which are achieved with the presented implementation.

\section{TanDEM-X Spotlight Interferometry}

TanDEM-X (TDX) is the twin satellite of TerraSAR-X (TSX), and the mission purpose of this constellation is to acquire a global homogeneous DEM [4]. Using InSAR to generate a DEM (digital elevation model) is well known since NASA's SRTM (Shuttle Radar Topography Mission). However, there are certain aspects that have to be considered to maximize the quality of the interferogram and therefore the resulting DEM. In order to minimize phase decorrelation (due to temporal changes or surface deformation), both satellites have to record the images within a very short time delay. Another aspect in DEM generation is the height ambiguity, which is determined by choosing an appropriate baseline. Both satellites orbit consistently in very close formation, recording images at almost the same time with a defined baseline. Images taken in this setup show very high coherence and are therefore particularly suitable for interferogram generation, as shown in Figure 15.

2.1. Mission Design for Bistatic Image Acquisition. The primary mission goal of TanDEM-X is the generation of a DEM of unprecedented accuracy with a spatial resolution of $12 \mathrm{~m}$ and a relative vertical accuracy of $2 \mathrm{~m}$ [16]. The global coverage will be larger towards the poles compared to the SRTM [16].

The mission design is a constellation of TSX and TDX as a twin concept. It is based on two almost identical SAR systems orbiting in close formation. When TSX was launched, it already incorporated all necessary equipment and procedures to operate with a second satellite. Both satellites need to be able to synchronize the phase; therefore, X-band horn antennas are installed on both satellites. The dual frequency GPS receiver estimates the orbit parameters precisely. The pulse repetition frequency (PRF) is synchronized by GPS measurements. An additional cold gas propulsion system 


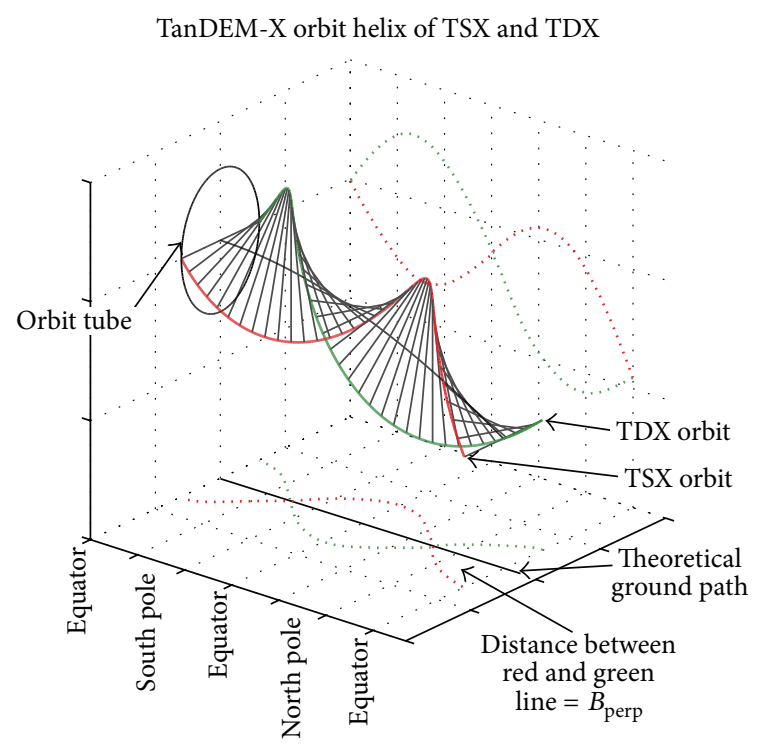

Figure 2: TerraSAR-X (red) and TanDEM-X (green) orbit constellation-close formation helix-like orbit. $X$-axis is one revolution around the globe.

allows TDX to keep the right orbit attitude. Both satellites can operate separately in the image mode spotlight (see image formation in Section 3.1), stripmap, or ScanSAR. Operating as a twin system brings up three different data acquisition modes these include, bistatic - which will be discussed below-pursuit monostatic, and alternating bistatic [17].

In bistatic imaging, one satellite is serving as transmitter, and both satellites receive the backscattered signal. Temporal decorrelation is minimized, and the atmospheric effects are equal in both images [4]. Ground objects that move within this velocity range, for example, water or trees affected by wind, are still incoherent. Coherent results are achieved by synchronizing the PRF between the two receiving antennas. The desired difference between both images is a slightly different view angle. That is expressed by the effective baseline, which is half the perpendicular baseline [4]. The modeling and determination of the orbital baseline for bistatic are discussed in [18].

To ensure a constant baseline, both satellites orbit in a helix-like fashion, with a short distance along the flight pass. One satellite rotates around the orbit tube of the second satellite. This is necessary to keep an effective baseline of around $200 \mathrm{~m}$ [4]. Schematic sketches are shown in Figures 2 and 3. One can imagine two circular orbits with a cross track distance of $\sim 360 \mathrm{~m}$ at the Equator and $\sim \pm 400 \mathrm{~m}$ radial distance at the poles [4]. With two orbits as described above, one satellite (e.g., TSX) is considered as the reference. The second spacecraft (e.g., TDX) is then revolving around the orbit of the reference. One whole circle is performed during one revolution around the Earth. Having a controlled orbit gives a predictable perpendicular baseline and height ambiguity.
The differences between repeat pass and single pass interferometry are visualized in Figure 3. Repeat pass interferometry refers to a single satellite system $(M)$ acquiring one image at a time. To observe the Earth two times in a row with similar image geometry-a prerequisite for interferometry [2] - the satellite has to pass the same location/orbit again. However, this is only possible after a certain amount of time, expressed by the temporal baseline, for example, $\sim 11$ days for TSX. Single pass interferometry refers to an image pair generation within the same overpass. The temporal baseline between both images is then nearly zero. The advantages of two satellites $(M$ and $S$ ) being just several hundred meter apart is that the temporal phase decorrelation is very low. A coherent image pair will produce an interferogram of high accuracy and quality with minimized temporal decorrelation due to vegetation or varying weather conditions. Atmospheric effects on two different dates can be neglected [4].

2.2. Processing Considerations. In this part, we will discuss the actual processing steps for TDM images necessary to generate an interferogram and subsequently flatten it. A bistatic complex image pair is the recorded backscatter signal of two identical satellite antennas with a defined baseline. The delivered TDM spotlight product is an already coregistered pair of standard SSC images and named CoSSC. Obligatory processing steps before interferogram formation are already done. This information is mentioned in the auxiliary XML file of a TDM CoSSC image pair. During the processing with DORIS, three issues occurred: (i) the changes in the data format to 16-bit floating point, (ii) extracting the right baseline, and (iii) the different range (travel) times for the reference phase.

TDM data are delivered in the CoSSC file format with complex half-precision float values (float16). Float16 is defined by the IEEE 754 standard and explained in [19]. SSC data is delivered as complex integer with 16-bit precision. As a result, one complex number consists of two 16-bit floating-point values. A conversion from float16 to float32 was performed with a library developed by [20]. Correct values are maintained, and the data can be used in non-float 16 compatible software.

The metadata states that both images are cropped to the same extent, corrected for bistatic mode, spectral filtered in range and azimuth, coregistered, and resampled. Therefore, interferogram generation can be started directly. Depending on the setup, either one of the satellites is used as the master (TSX or TDX). By taking the complex phase information of the master and slave, an interferogram can be derived with

$$
I=M \cdot S^{*} \cdot R^{*}
$$

$M$ is the complex signal of the master, $S^{*}$ is the complex conjugate of the slave, $R^{*}$ the conjugate of the reference phase, and $I$ is the resulting interferogram [21].

DORIS extracts the orbital parameters slightly different for TDM. By default, it reads the data as if both images were acquired independently in the monostatic mode. Bistatic image processing with TDM data is not considered [15]. The 


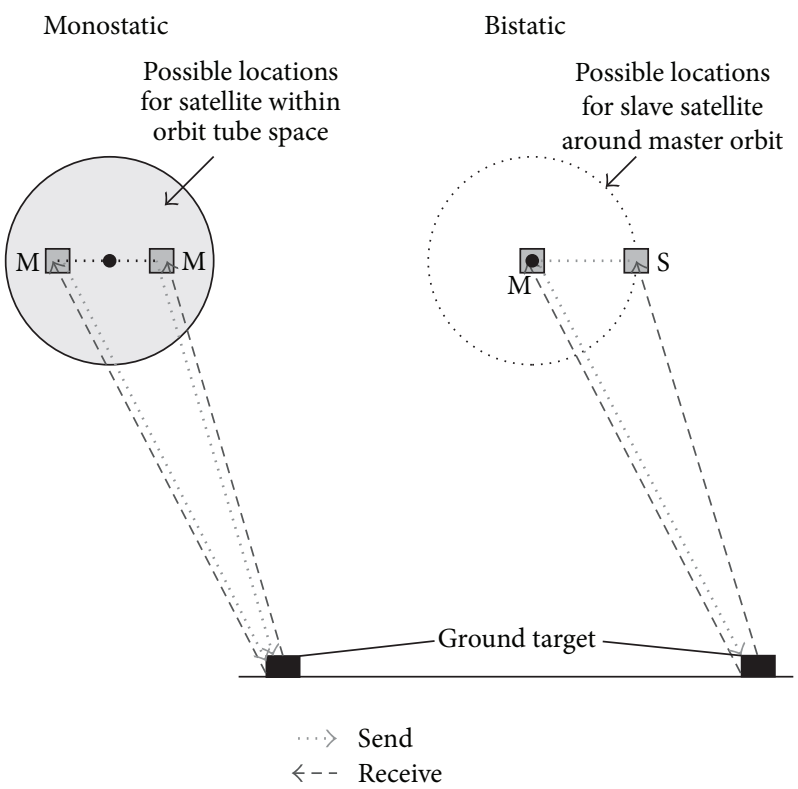

FIGURE 3: Monostatic (left side) image acquisition on two dates: the satellite $(M)$ sends a signal (dotted line) and receives the echo (dashed line) during each overpass. Bistatic image acquisition generates two images in a single overpass with almost no time difference. One satellite serves as transmitter, and both serve as receiving antennas. The baseline of the slave satellite is desired to be on a circle around the master, see Figure 2.

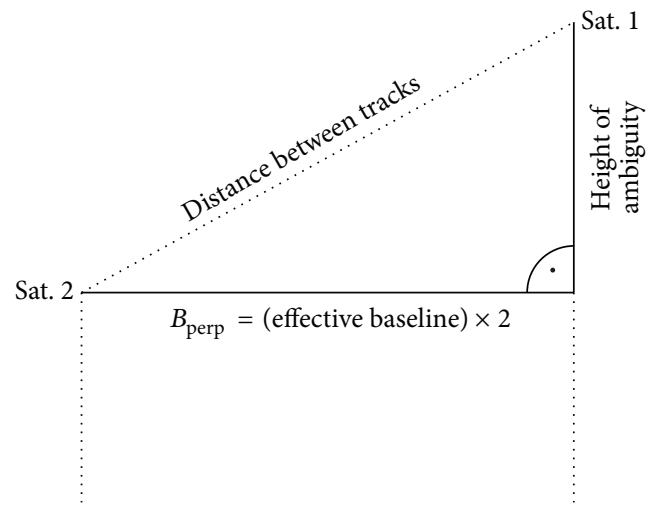

FIGURE 4: Relation of baseline annotations and orbit geometry for TanDEM-X.

orbit information is retrieved based on the annotation files of TSX and TDX separately. Both files are treated as if they were two separate acquisitions with a temporal baseline of 0 days. This is sufficient at the initial reading step. When estimating the baseline in DORIS, a mismatch between the perpendicular baseline and the effective baseline occurred. According to Krieger and Moreira [4], the effective baseline is half the length of the perpendicular baseline which is due to the bistatic acquisition mode (see Figure 4). The DORIS result file has to be modified accordingly.

Due to the bistatic acquisition, one has to take care of the range times by taking half the travel time instead of the full range, distance from and to the antenna. The reference phase $\Phi_{\text {reference }}$ as mentioned and annotated with $R$ in (1) for a mono- or bistatic image pair is calculated by

$$
\begin{aligned}
\Phi_{\text {reference }}= & \Phi_{\text {Master_const }_{\text {s.range }}} \cdot \mathbf{t}_{\text {Master }} \\
& -\Phi_{\text {Slave }_{\text {const }}} \cdot \mathbf{t}_{\text {range Slave }} .
\end{aligned}
$$

$\Phi_{\text {Master_const }}$ is a constant value, based on the wavelength of the master image and derived with either option in

$$
\begin{aligned}
& \text { Monostatic : }\left\{\Phi_{\text {Master_const }}=\frac{-4.0 \cdot \pi \cdot c}{\lambda_{\text {Master }}}\right\}, \\
& \text { Bistatic : }\left\{\Phi_{\text {Master_const }}=\frac{-2.0 \cdot \pi \cdot c}{\lambda_{\text {Master }}}\right\},
\end{aligned}
$$

where $c$ is the speed of light and $\lambda_{\text {Master }}$ is the wavelength of the master image. For a signal traveling to the ground and back to the satellite (monostatic), the factor would be -4.0 , for two full phase circles, instead of -2.0 . This comes from the radar range equation and has to be changed for the bistatic mode. $\mathbf{t}_{-}$range $\mathrm{Master}_{\mathrm{m}}$ is the range time based on the pixel location as stated as follows:

$$
\mathbf{t}_{-} \text {range } \text { Master }=\mathbf{t}_{-} \text {range }_{0}+\frac{(\text { pixel }-1.0)}{\text { RSR }} \text {. }
$$

Pixel is the pixel number (column as integer) of that particular line, and RSR is the range sampling rate as annotated in the metadata. The interferometric phase can now be processed correctly.

\section{TerraSAR-X Spotlight Interferometry}

Very high-resolution spaceborne SAR images generated with current satellites are only possible by sweeping the 


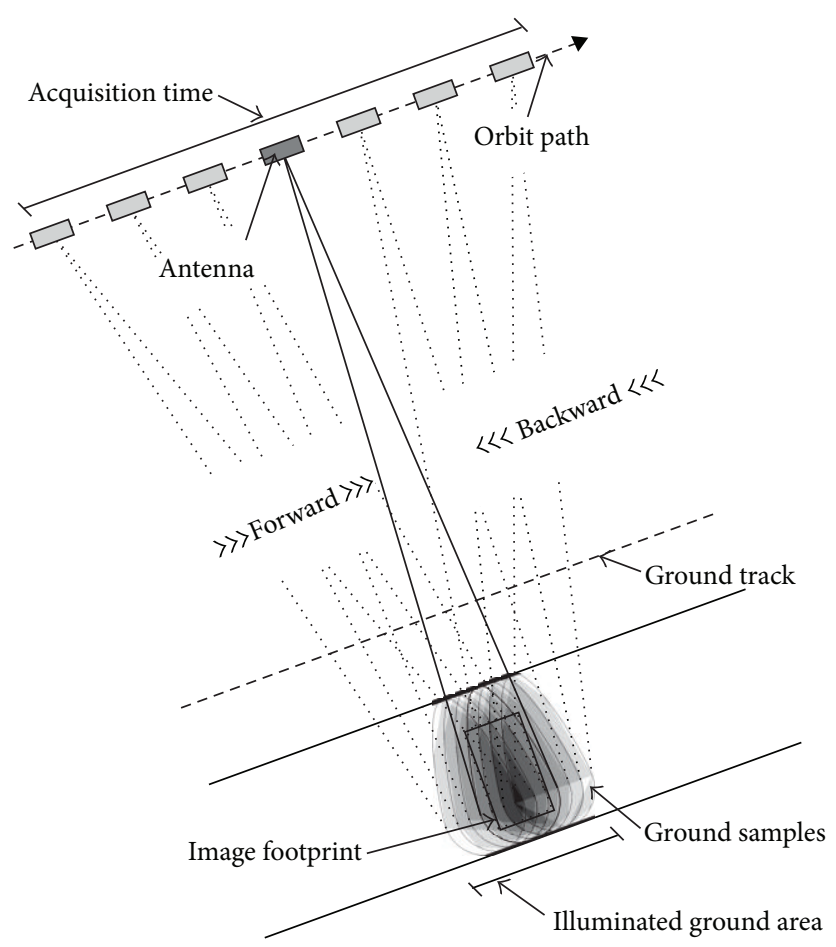

FIGURE 5: Image acquisition geometry for spotlight mode. The beam is steered backwards when the satellite passes the area of interest [4].

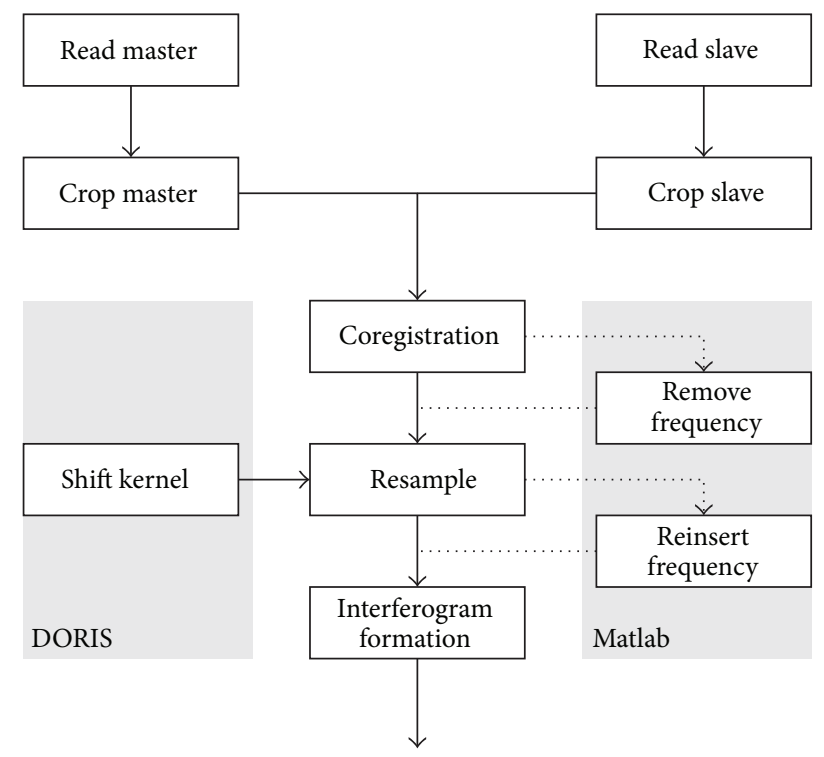

FIGURE 6: General processing flow chart. Two options: implementation in DORIS shifting the kernel (left); external scripts in MATLAB to set the image spectrum to zero (right).

antenna beam in spotlight mode. The occurring problems are mentioned above. We argue that especially high-resolution interferograms would be explored more frequently, if the processing could be done with more ease.

3.1. Spotlight Image Formation in Repeat Pass. Spotlight image mode of TSX and TDX is a sliding azimuth beam

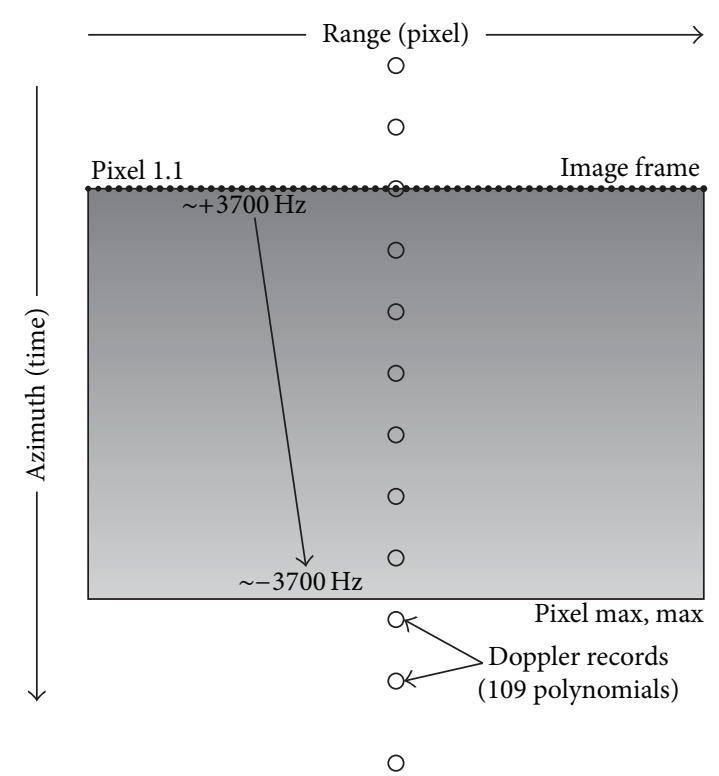

FIGURE 7: Schematic sketch of interpolation values derived from XML data. The $x$-axis represents the range time calculated from the pixel number, and the $y$-axis is interpolated from the annotated Doppler polynomial records. The recording of them starts and ends before and after the actual image footprint.

steering technique [14]. As shown in Figure 5, the satellite passes the area of interest in a certain time. The observation time is increased by steering the antenna beam in azimuth. See Figure 5 for a diagram of this process. The drift rate of 


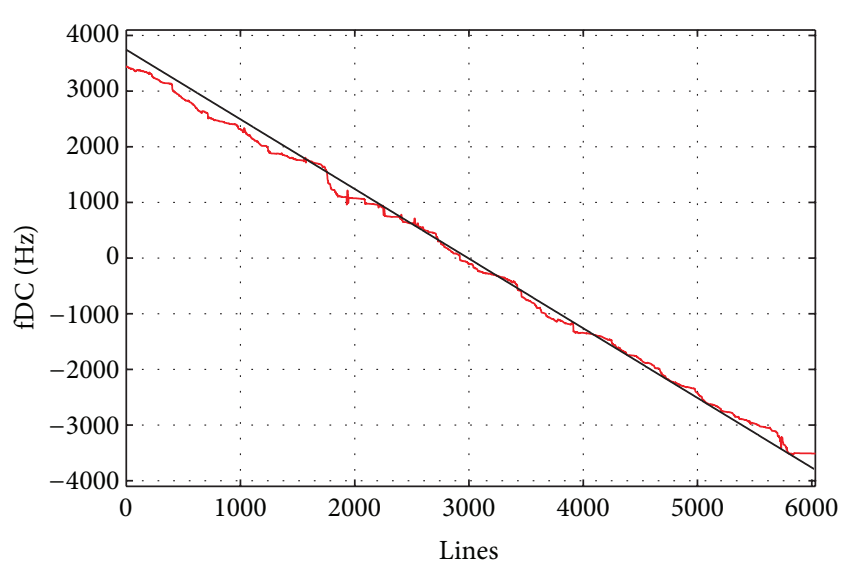

- Actual frequency

— Modeled frequency

Figure 8: Modeled and actual Doppler frequency at pixel 4000 (range) from the first to the last line.

the sweeping motion is slower than the overpass velocity. Consequently, the beam is sliding over an area and not starring at a single location. This results in more ground samples and an increased ground resolution. One shortcoming is the limited image frame length. A longer illumination time is achieved in azimuth, but not in range. High-range resolution is accomplished with a bandwidth of $300 \mathrm{MHz}$ [13].

The Doppler frequency decreases over the whole image length in azimuth. This behavior is different compared to the standard stripmap mode. The Doppler spectrum of a spotlight image follows a linear trend from positive Doppler frequencies around $3700 \mathrm{~Hz}$, to negative values around $-3700 \mathrm{~Hz}$, as visualized in Figure 7. Theoretically, only one zero Doppler line exists in the center of the image. This is the case when the beam is steered exactly perpendicular to the orbit and the Earth. Stripmap images do not have this linear trend-all lines are centered around the Doppler frequency. Proper treatment of the Doppler frequency is necessary for InSAR processing. The amplitude information is not affected.

3.2. Implementation of Processing Method. As discussed in $[14]$ and mentioned in $[15,22]$, there are two options to deal with the different image spectra of master and slave. Correct phase information is kept when the spectrum of the slave is set to zero before resampling or the interpolation kernel is shifted during the resampling step. We implemented the first option in MATLAB. Both options are shown as a flow chart in Figure 6. Shifting the interpolation kernel is the second option and is described in detail in [14]. The according Doppler frequency, to multiply the kernel with, is retrieved by exploiting the annotated Doppler polynomials (see Figure 7). Eineder et al. [14] propose a low-pass azimuth bandpass filter after resampling. We will focus solely on the generation of the frequency matrix for the pixel-by-pixel calculation of the interpolation kernel.

The chosen interpolation kernel is a six-point cubic convolution kernel as described in [22], implemented in DORIS and shown in (5). Hanssen and Bamler [22] recommend a six-point cubic convolution kernel for high-resolution applications that is also recommended by Eineder et al. [14] and used in our implementation:

$$
i(x)=\left\{\begin{array}{c}
(\alpha-\beta+2)|x|^{3}-(\alpha-\beta+3)|x|^{2}+1 \\
0 \leq|x|<1 \\
\alpha|x|^{3}-(5 \alpha-\beta)|x|^{2}+(8 \alpha-3 \beta)|x|-(4 \alpha-2 \beta) \\
\beta|x|^{3}-(8 \beta)|x|^{2}+(21 \beta)|x|-(18 \beta) \\
0 \\
0 \leq|x|<3 \\
3 \leq|x|,
\end{array}\right.
$$

where $\alpha$ and $\beta$ are two parameters $(\alpha=-1 / 2$ and $\beta=1 / 2)$ as defined by [22]. The resulting interpolation kernel $i(x)$ is then multiplied with the modeled Doppler frequency during resampling.

To calculate the frequency matrix, the timing and Doppler rate metadata are evaluated for each product specifically. See appendix of $[14,23]$ for a more elaborate description. Figure 7 helps to visualize the pixel-by-pixel calculation for the frequency matrix. The number of Doppler polynomial records is variable and not fixed. The recording of these discrete measurements starts and ends before and after the actual image footprint.

Each annotated Doppler polynomial is evaluated along the range time as described in [16] and shown as follows:

$$
\begin{aligned}
\mathrm{fDC}(\text { pixel })= & \left(\mathrm{fDC}_{\operatorname{coef} f 0} *(P(\text { pixel })-\text { Refpoint })^{0}\right) \\
& +\left(\mathrm{fDC}_{\operatorname{coef} f 1} *(P(\text { pixel })-\text { Refpoint })^{1}\right) \\
& +\left(\mathrm{fDC}_{\operatorname{coef} f 2} *(P(\text { pixel })-\text { Refpoint })^{2}\right) .
\end{aligned}
$$

$P$ (pixel) is the function pixel $2 t_{\text {range }}$ (pixel) which calculates the range time based on the pixel number (integer value), the range sampling frequency RSF, and the start range time $t_{\text {range } 0}$. The corresponding calculation is shown as follows:

$$
\text { pixel } 2 t_{\text {range }}(\text { pixel })=t_{\text {range } 0}+\frac{(\text { pixel }-1.0)}{\text { RSF }}
$$

The timing information gathered from the metadata are in the system receive time (the actual uptime of the satellite) and has to be converted to zero-Doppler time system by

$$
t_{\mathrm{SSC}}=\left(t_{\mathrm{RAW}}-\left(\frac{f_{\mathrm{DC}}\left(t_{\mathrm{RAW}}\right)}{F M_{\text {rate }}}\right)\right)-t_{\text {zero-Doppler Start }} \text {. }
$$

$t_{\text {RAW }}$ is the time UTC annotation in seconds, and $t_{\text {zero-Doppler Start }}$ is the actual first zero Doppler time. The mentioned $F M_{\text {rate }}$ is calculated according to the following function

$$
F M_{\text {rate }}=\frac{\dot{\mathrm{fDC}} \mathrm{Coef} f 0-\dot{\mathrm{fDC}} \mathrm{Coef} f 1}{2} .
$$




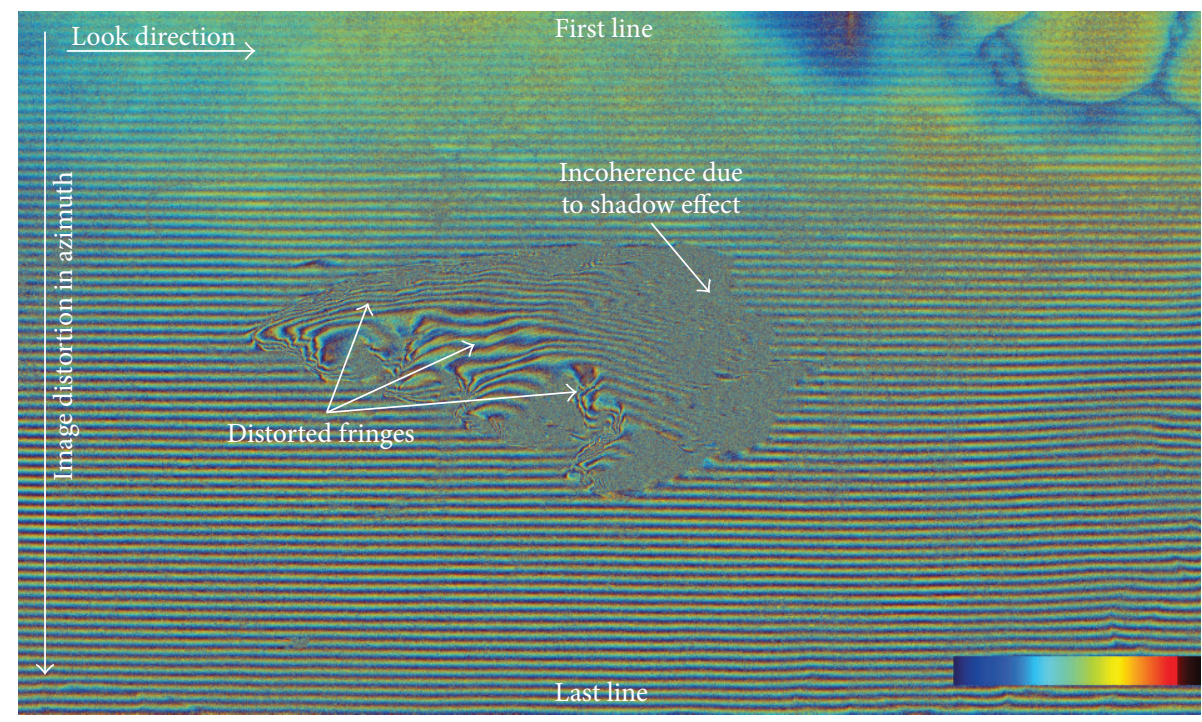

FIGURE 9: TerraSAR-X image pair (Uluru) with default frequency shift in DORIS. The image is not corrected and gets distorted towards the last line.

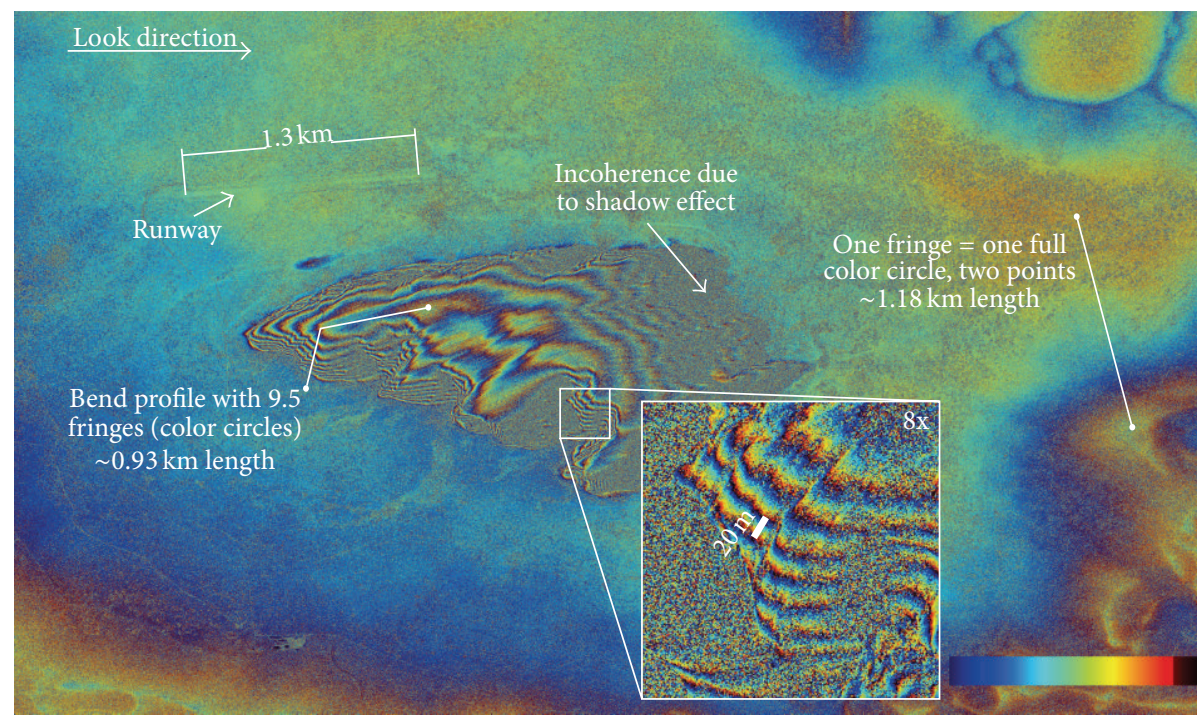

FIGURE 10: Interferogram generated with DORIS using the cc6p_SP interpolation kernel. Temporal baseline is 11 days and perpendicular baseline is $233.7 \mathrm{~m}$ with a height ambiguity of $34 \mathrm{~m}$. Master acquired on 2009-02-12 and slave on 2009-02-23.

$\mathrm{fDC}_{\text {coef } f 0}$ marks the Doppler rate at the start of the scene and $\mathrm{fDC}_{\text {coef } f 1}$ at the end of the scene. The $F M_{\text {rate }}$ is the average of these values. To interpolate along azimuth, the fDC and $t_{\mathrm{SSC}}$ values are used.

Comparing the actual frequency and the interpolated modeled frequency derived from the metadata is shown in Figure 8. The spectrum for a single line in azimuth is colored in red and follows an almost linear trend from approx. $3500 \mathrm{~Hz}$ in the first line to around $-3500 \mathrm{~Hz}$ in the last line of the image. To shift the kernel, the modeled frequency was used (black line).

\section{Results and Discussion}

Results for one bistatic TDM product and several monostatic image pairs from TSX and TDX have been produced. A TDM pair from Las Vegas in high-resolution spotlight mode was used to test the bistatic configuration of DORIS, and 

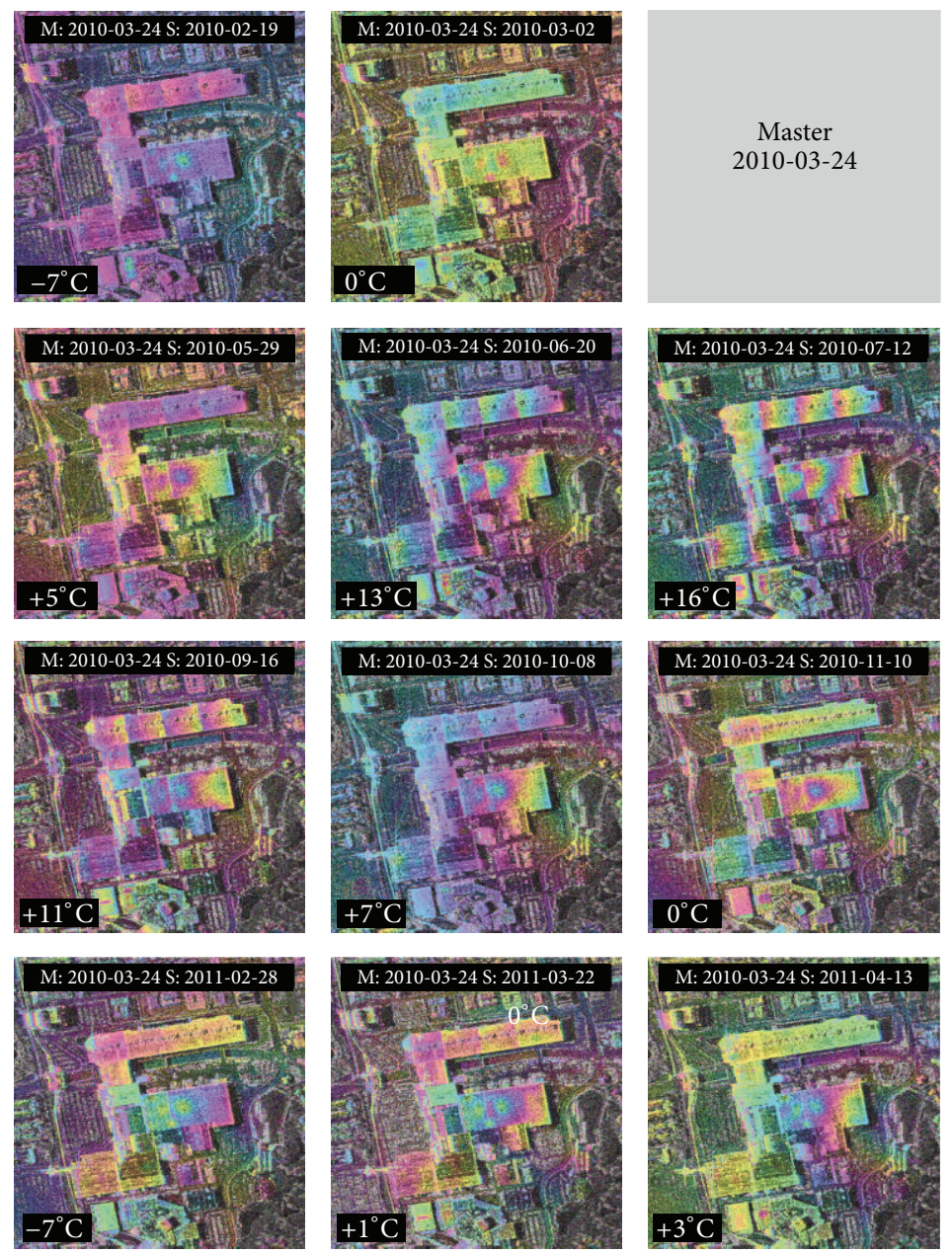
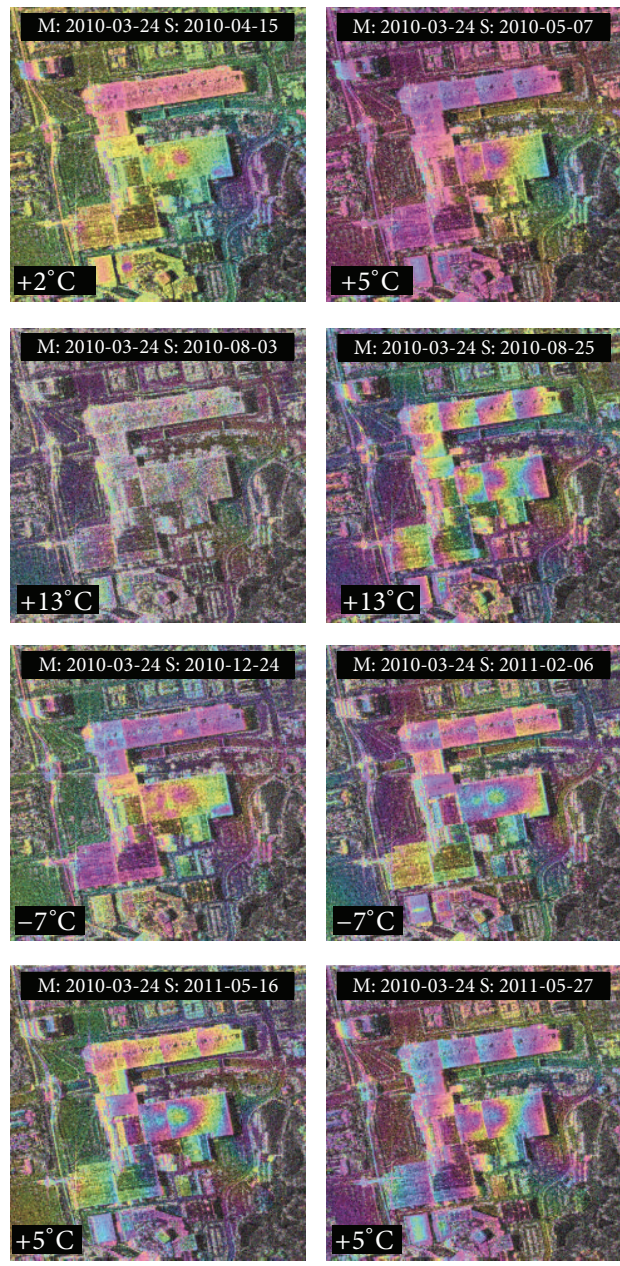

FIgURE 11: Time series of TerraSAR-X ascending stack, showing flattened interferograms. Area: Convention Center in Las Vegas, USA. Temperature annotations indicate the relative temperature difference to the master image (2010-03-24), source [18].

the result is shown in Figure 13. Our implementation of the cc6p_SP processing option in the resampling stage of DORIS (version 4.06 beta) was tested with the following TerraSAR$\mathrm{X}$ HS source images: ascending and descending data stacks from Las Vegas (20 images each) and one data stack from Lüneburg (16 images). In addition, a sample dataset from Uluru, Australia, provided by Infoterra Germany [24] was used. All scenes were acquired in high-resolution spotlight mode. The process chain includes all steps listed in DORIS manual [21] until coherence estimation. We present flattened interferograms, flattened and filtered interferograms, and coherence images.

The implementation of bistatic image processing was done in DORIS, and the results are shown in Figures 13 and 15. By taking care of the float16-bit data format, the reference phase, and the effective baseline, a coherent interferogram was generated.

To demonstrate the effect of running DORIS with the default parameters in the input card "resample", that is, frequency shift in azimuth as described in [21] during interpolation leads to the results shown in Figure 9. Applying the default frequency shift, results in an interferogram where the phase seems to be correct in the first lines, getting more and more distorted and corrupted towards the last line. This is due to the not applied linear kernel shift in azimuth. Using a six-point cubic convolution kernel with a linear frequency shift, as described above and in [21], yields a flattened interferogram as shown in Figure 10.

There are a few things to pay attention to in Figure 10, for example, fringes along steep slopes and distorted fringes due to low coherence caused by shadow effects. The results are not DEM corrected. The phase follows the topography. Along the bend profile (bottom to top of the rock), 9.5 fringes can be counted. Evidence for a relatively flat surrounding area is given by the distance between two points $(\sim 1.18 \mathrm{Km}$, annotation on the right) with one phase circle.

Stacking images is a commonly used technique for time series analysis. A TSX ascending image stack over Las Vegas was used to generate interferograms, flattened the phase, and then cropped to the extend of the Convention Center 

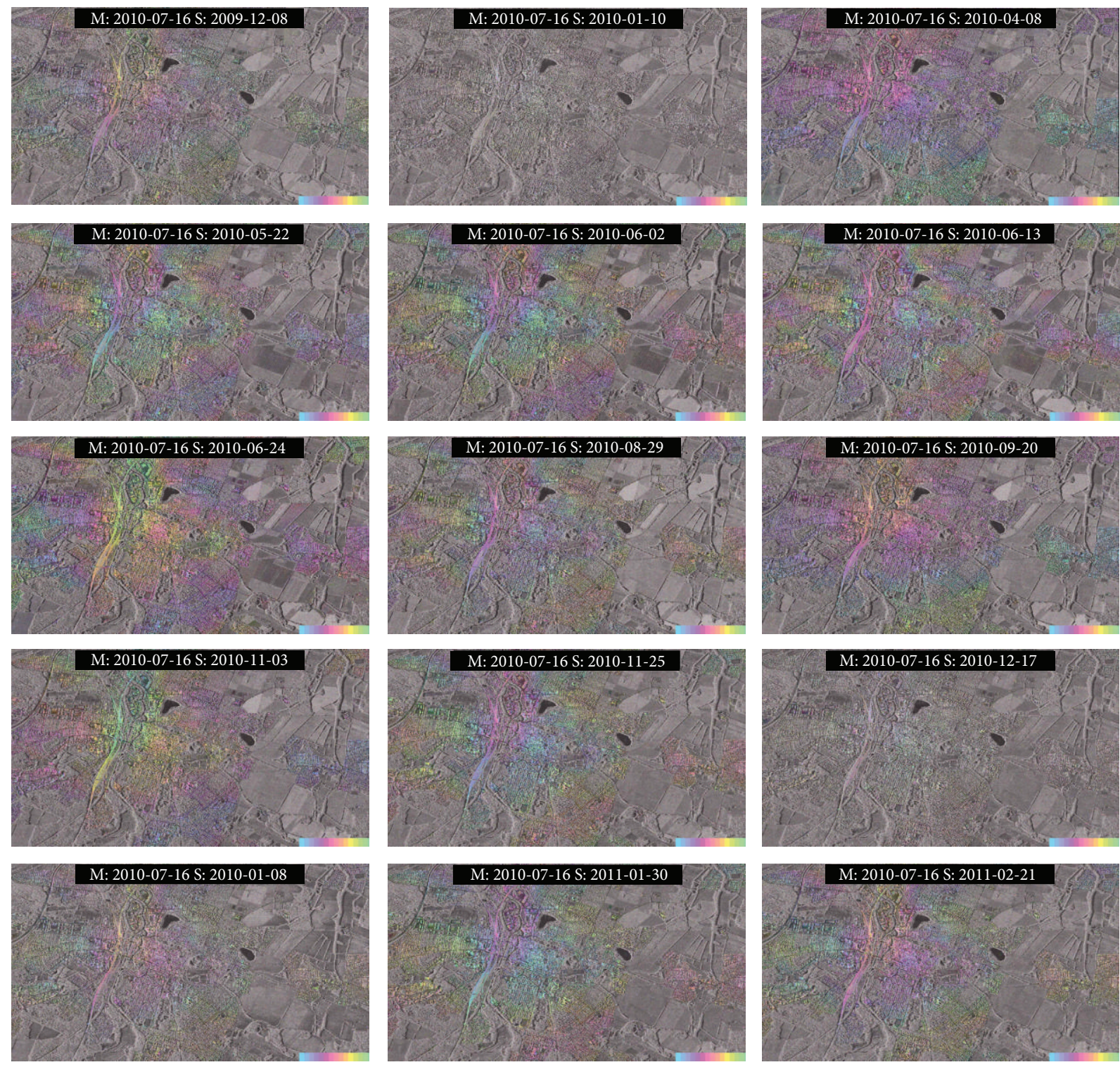

FIGURE 12: Time series of 15 full scene TerraSAR-X spotlight interferograms over Lüneburg, Germany. The color intensity has been increased extremely.

$\left(36^{\circ} 07^{\prime} 52.44^{\prime \prime}\right.$ North, $115^{\circ} 09^{\prime} 04,85^{\prime \prime}$ West), see Figure 11. Each pair was processed with exactly the same parameter set. The master image was chosen based on minimizing $B_{\text {perp }}$. The subsets are still in radar coordinates and not reprojected, the illumination direction is from the left. We show this result, because (a) the roof is a relatively flat surface with high backscatter, and (b) it is from the center of the image where the Doppler shift between both acquisitions is rather low. Relating the visualized phase to the temperature information (relative to the master), one can see that the roof gets more deformed the greater the absolute temperature difference.

The data stack of Lüneburg with 16 images shows less coherence. The interferograms for a full scene are depicted in Figure 12. Coherent areas are found in the urban area where ground is covered with solid structures like buildings and roads. The surrounding fields are mostly incoherent, and therefore homogeneous phase information cannot be derived for these areas. The master image was selected based on minimizing temporal and perpendicular baselines.

A comparison of bi- and monostatic images is illustrated in Figures 13 and 14. The higher brilliance of the TDM image pair, compared to the TSX/TDX image pair, is due to the small temporal decorrelation for bistatic image acquisitions.

Looking at the hotels and the Convention Center in Figures 13 and 14 demonstrates two things. First, the phase or fringes following along the front of the hotels are similar in both scenes. Second, the phase on the Convention Center is very smooth in the TDM image pair. On the other hand, the TSX image pair shows more fringes on the roof, indicating a motion of the roof in LOS (line of sight) between both acquisitions. 


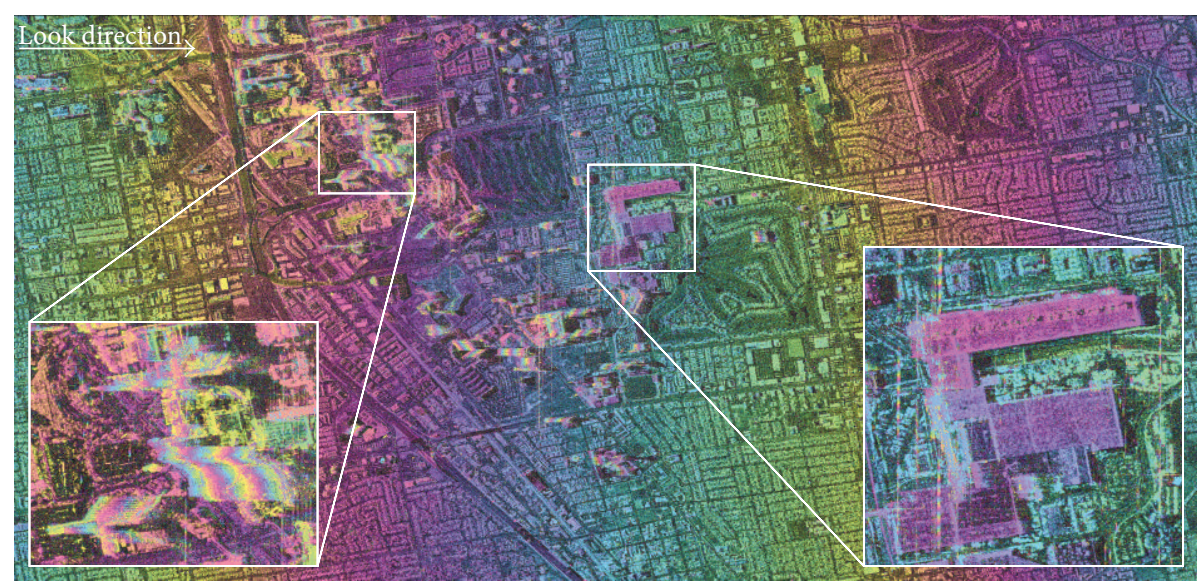

FIGURE 13: TanDEM-X flattened interferogram in radar coordinates with amplitude image in background. Subsets show hotels and the Las Vegas Convention Center. Temporal baseline 0 days, effective baseline: $\sim 87 \mathrm{~m}$.

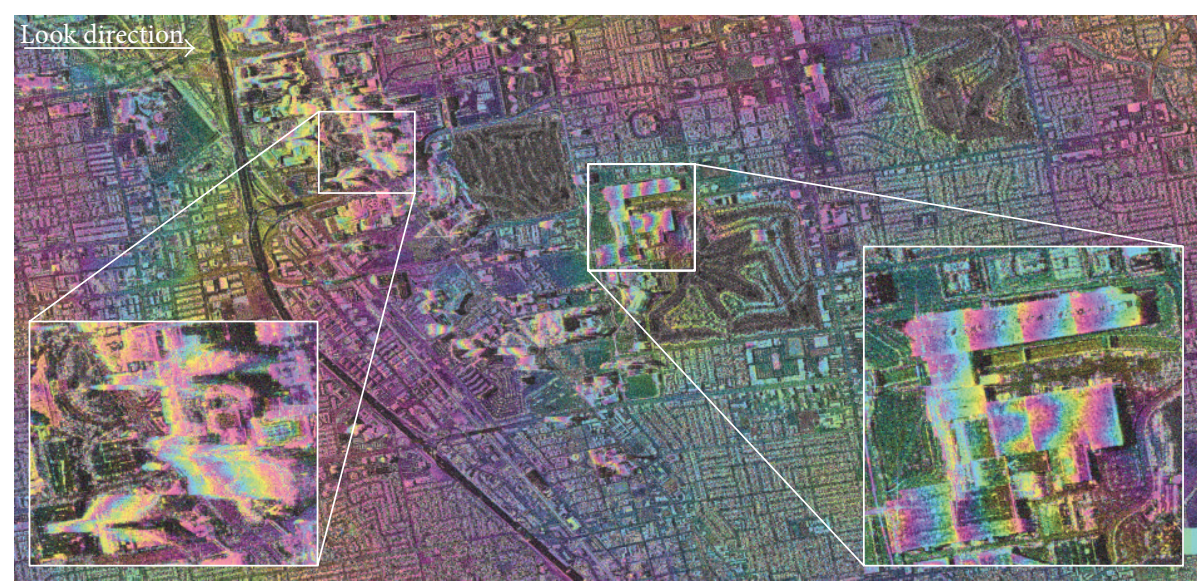

FIGURE 14: TerraSAR-X flattened interferogram in radar coordinates with amplitude image in background. Subsets show hotels and the Las Vegas Convention Center. Temporal baseline 110 days, perpendicular baseline: $48.1 \mathrm{~m}$.

On the subject of coherence-which is a strong indicator for the quality of an interferogram-the effect of bistatic acquisitions compared to repeat pass interferometry becomes apparent. In the bistatic TDM image pair over Las Vegas, we observe a mean coherence for the whole scene of about 0.7048 , see Figure 15. However comparing this with a pair of TerraSAR-X acquisitions with a temporal baseline of 11 days gives a mean coherence of 0.6486 , see Figure 16 . The mentioned pair was taken on 2010-09-19 for the master and 2010-09-08 for the slave. Picking two images with a longer temporal baseline of 110 days for 2010-03-24 (master) and 2010-07-12 (slave) gives a mean coherence of 0.5206 , see Figure 17 for a histogram as well.

Comparing the relatively dense urbanized scene of Las Vegas with a relatively rural setting in Lüneburg illustrates the following. The coherence for any scene in Lüneburg is rather low, for example, a temporal baseline of 22 days in summer between the images taken on the 2010-07-16 and 2010-0624 gives a mean coherence of 0.4029 . There are two special cases: one in Las Vegas and one in Lüneburg where very low coherence was noticeable by visual interpretation. The image pairs in question are 2010-03-24 with 2010-08-03 (Las Vegas) and 2010-07-16 with 2010-01-10 (Lüneburg) which show a mean coherence value of 0.4071 and 0.3506 , respectively. In the case of Lüneburg we were able to retrace the possible cause by checking the weather data, that was snowfall [25] on that particular day.

\section{Conclusion}

In this paper, we explained the steps necessary to process bistatic and monostatic TanDEM-X and TerraSAR-X image acquisitions utilizing a public domain tool: DORIS. DORIS was modified and extended for this purpose. We have also clarified the basic concepts of bistatic acquisitions and illustrated linear Doppler drift, which occurs during the beam steering technique used in spotlight image creation. We specifically focused on the generation of a Doppler frequency matrix that is used to shift the interpolation kernel during 


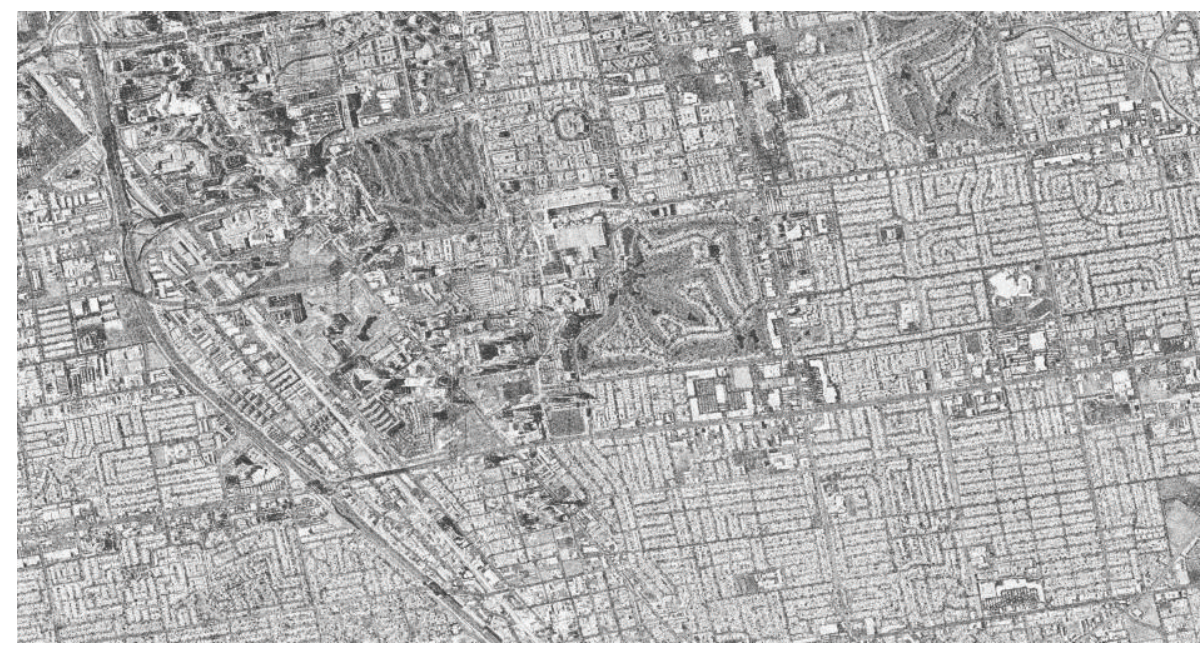

Figure 15: TanDEM-X coherence image. The brighter the more coherent. Parks and water become visible due to the effects of wind.

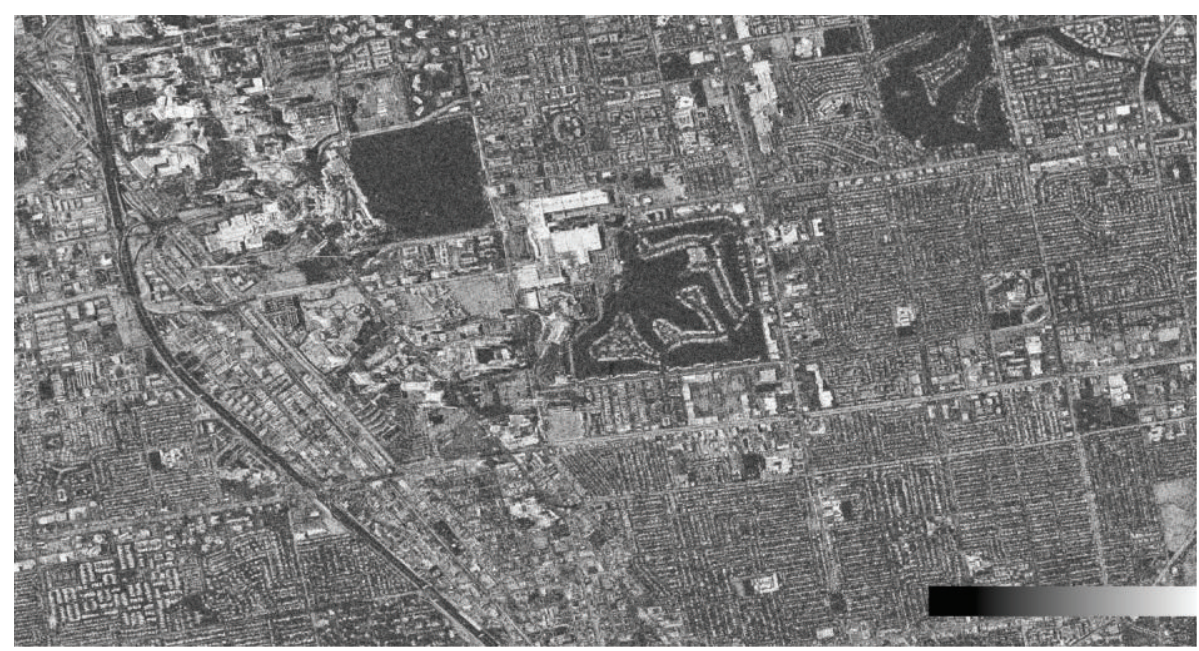

FIgURE 16: TerraSAR-X repeat pass coherence image. Master 2010-07-12 slave: 2010-03-24.

resampling. Furthermore experimental results based on several input datasets, demonstrated that our implementation gives consistently good output. A comparison of bistatic TDM and monostatic TSX/TDX interferograms shows the difference in phase and general coherence.

The character of the paper is rather instructional with a focus on the explanation of bi- and monostatic very highresolution SAR images. The key point lies within the guidance to understand and produce TSX spotlight interferograms. We also show that advanced image acquisition techniques like spotlight and or bistatic spotlight can be processed with already available open-source software solutions. Additionally, it demonstrates the quality and consistency of the data which has been used in the experiments. This paper can serve as a basis for future papers by illustrating the potential of the very high-resolution repeat or single pass SAR interferometry.

A TanDEM-X interferogram can be retrieved by just a few modifications in the source code of DORIS. The overall coherence of the image is relatively high, given the very short temporal baseline in bistatic acquisitions. Experimental results showed that the roof of the Las Vegas Convention Center or other buildings can exhibit thermal deformation. This is observable due to the phase information. In the case of TDM, the phase is very homogenous because of the contemporaneous image acquisition. That indicates no motion, at least not detectable with SAR within the acquisition period.

Particularly interesting is the comparison of the bistatic and monostatic images. The overall coherence of a TDM is, as expected, very high. Even water does not show up with the significant dark signature. The TSX image pair with 110 days temporal baseline is less coherent, and objects in quick motion like trees/leafs and water show very low coherence.

Spotlight InSAR in DORIS requires some modifications to calculate consistent interferograms. The annotated Doppler information in the XML metafile have to be evaluated, and a Doppler frequency matrix was created. It was then used to shift the six-point cubic convolution kernel 


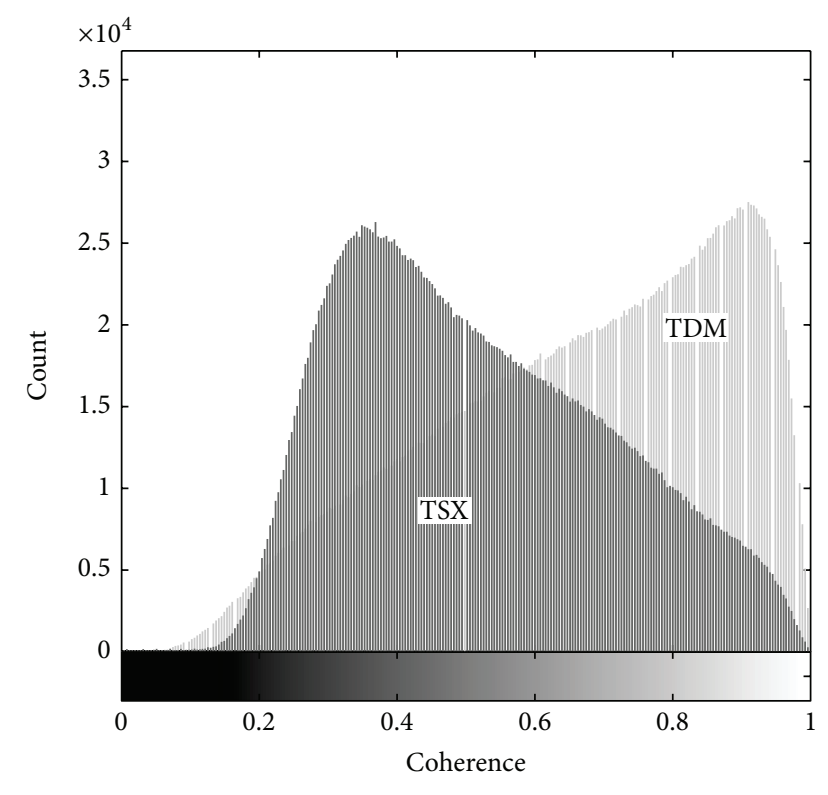

FIGURE 17: Coherence histogram comparison between TerraSAR-X (TSX) with 110 days $B_{\text {temp }}$ and a TanDEM-X product (TDM).

during the resampling stage to match both image spectra. A new "cc6p_SP" option in the processing input card "RS_METHOD" in DORIS has been added to accomplish this. We have compared the result to a calculation that used the default kernel shift in DORIS. The result was expectantly distorted. Additionally, we compared to a result made by a commercially available software, with very high agreement to our result.

Interpreting the phase information on the roof of the Las Vegas Convention Center gave evidence of correlation between temperature and number of fringes or distance between fringes, respectively. The higher the temperature differences to the master image the greater the deformation. This was qualitatively assessed by looking at the fringes appearing for separate roof sections.

The initial goal was to provide an implementation of an already known algorithm to process TerraSAR-X spotlight images in freely available software, DORIS. As seen from the results, the integrated processing option to shift the interpolation kernel according to the Doppler frequency works seamlessly and effectively. It enables researchers to process high-resolution spotlight interferograms.

The code is directly available upon request from the corresponding author and will probably be included in the next release of DORIS.

\section{Acknowledgments}

This work is financially supported by the National Key Basic Research Program of China (Grant no. 2013CB733205), the National Natural Science Foundation of China (Grant no. 41174120), and the German Academic Exchange Service (DAAD). The authors would like to thank the German
Aerospace Center (DLR) for providing the test datasets via the DLR AO LAN1301 and LAN0634.

\section{References}

[1] M. Eineder, H. Runge, E. Börner et al., "SAR interferometry with TerraSAR-X," in Proceedings of FRINGE Workshop, Frascati, Italy, December 2003.

[2] R. Hanssen, Radar Interferometry: Data Interpretation and Error Analysis, 2001.

[3] I. Woodhouse, Introduction to Microwave Remote Sensing, 2005.

[4] G. Krieger and A. Moreira, "TanDEM-X: a satellite formation for high-resolution SAR interferometry," IEEE Transactions on Geoscience and Remote Sensing, vol. 45, no. 11, pp. 3317-3341, 2007.

[5] U. Soergel, U. Thoennessen, A. Brenner, and U. Stilla, "Highresolution SAR data: new opportunities and challenges for the analysis of urban areas," IEE Proceedings: Radar, Sonar and Navigation, vol. 153, no. 3, pp. 294-300, 2006.

[6] P. Gamba, B. Houshmand, and M. Saccani, "Detection and extraction of buildings from interferometric SAR data," IEEE Transactions on Geoscience and Remote Sensing, vol. 38, no. 1, pp. 611-618, 2000.

[7] T. Balz and M. Liao, "Building-damage detection using postseismic high-resolution SAR satellite data," International Journal of Remote Sensing, vol. 31, no. 13, pp. 3369-3391, 2010.

[8] Y. Dong, Q. Li, A. Dou, and X. Wang, "Extracting damages caused by the $2008 \mathrm{Ms} 8.0$ Wenchuan earthquake from SAR remote sensing data," Journal of Asian Earth Sciences, vol. 40, no. 4, pp. 907-914, 2011.

[9] D. Ehrlich, H. D. Guo, K. Molch, J. W. Ma, and M. Pesaresi, "Identifying damage caused by the 2008 Wenchuan earthquake from VHR remote sensing data," International Journal of Digital Earth, vol. 2, no. 4, pp. 309-326, 2009.

[10] T. Moon, I. Joughin, B. Smith, and I. Howat, "21st-century evolution of greenland outlet glacier velocities," Science, vol. 336, no. 6081, pp. 576-578, 2012.

[11] D. Perissin, Z. Wang, and H. Lin, "Shanghai subway tunnels and highways monitoring through Cosmo-SkyMed Persistent Scatterers," ISPRS Journal of Photogrammetry and Remote Sensing, vol. 73, pp. 58-67, 2012.

[12] T. Wang and D. Perissin, "Deformation monitoring by long term D-InSAR analysis in Three Gorges area, China," IEEE Geoscience and Remote Sensing Letters, vol. 4, pp. 7-10, 2008.

[13] German Aerospace Agency, DLR, "TerraSAR-X ground segment basic product specification document," in Working Paper TX-GS-DD-3, no. 1.7, 2010.

[14] M. Eineder, N. Adam, R. Bamler, N. Yague-Martinez, and H. Breit, "Spaceborne spotlight SAR interferometry with TerraSAR-X," IEEE Transactions on Geoscience and Remote Sensing, vol. 47, no. 5, pp. 1524-1535, 2009.

[15] B. Kampes, R. Hanssen, and Z. Perski, "Radar interferometry with public domain tools," in Proceedings of FRINGE Workshop, Frascati, Italy, December 2003.

[16] H. Fiedler, G. Krieger, M. Werner, and K. Reiniger, "The TanDEM-X mission design and data acquisition plan," in Proceedings of the European Conference on Synthetic Aperture Radar (EUSAR '06), Dresden, Gemany, May 2006.

[17] German Aerospace Agency, DLR, "TanDEM-X ground segment experimental product description," in Working Paper TDGS-PS-3028, no. 1.2, 2007. 
[18] J. Tu, D. Gu, Y. Wu, and D. Yi, "Error modeling and analysis for InSAR spatial baseline determination of satellite formation flying," Mathematical Problems in Engineering, vol. 2012, pp. 123, 2012.

[19] J. van der Zijp, "Fast half float conversions," Working paper, 2012ftp://www.fox-toolkit.org/pub/fasthalffloatconversion.pdf.

[20] C. Rau, "Half-precision floating point library," sourceforge.net, 2012, http://half.sourceforge.net/.

[21] Technical University Delft, "Delft Object-oriented Radar Interferometric Software User's manual and technical documentation," Tech. Rep., Delft, The Netherlands, 2008.

[22] R. Hanssen and R. Bamler, "Evaluation of interpolation kernels for SAR interferometry," IEEE Transactions on Geoscience and Remote Sensing, vol. 37, no. 1, pp. 318-321, 1999.

[23] German Aerospace Agency, DLR, "TerraSAR-X ground segment level $1 \mathrm{~b}$ product format specification," in Working Paper TX-GS-DD-3307, no. 1.3, 2007.

[24] ASTRIUM, "Sample Imagery, Australia, Urulu-InSAR," 2009, http://www.astrium-geo.com/en/23-sample-imagery.

[25] The Weather Channel Companies, "Weather Underground," 2010, http://www.wunderground.com/. 

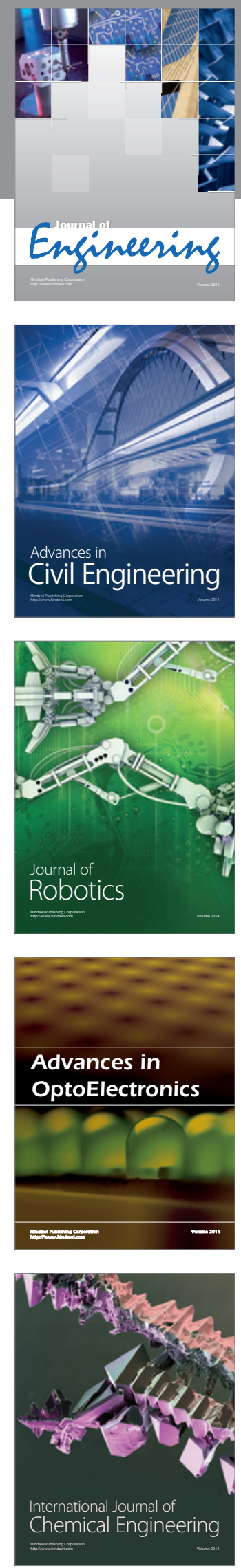

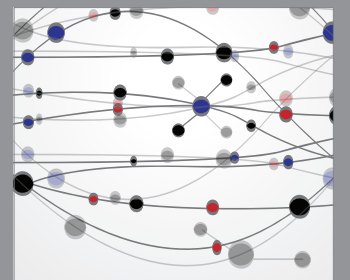

The Scientific World Journal
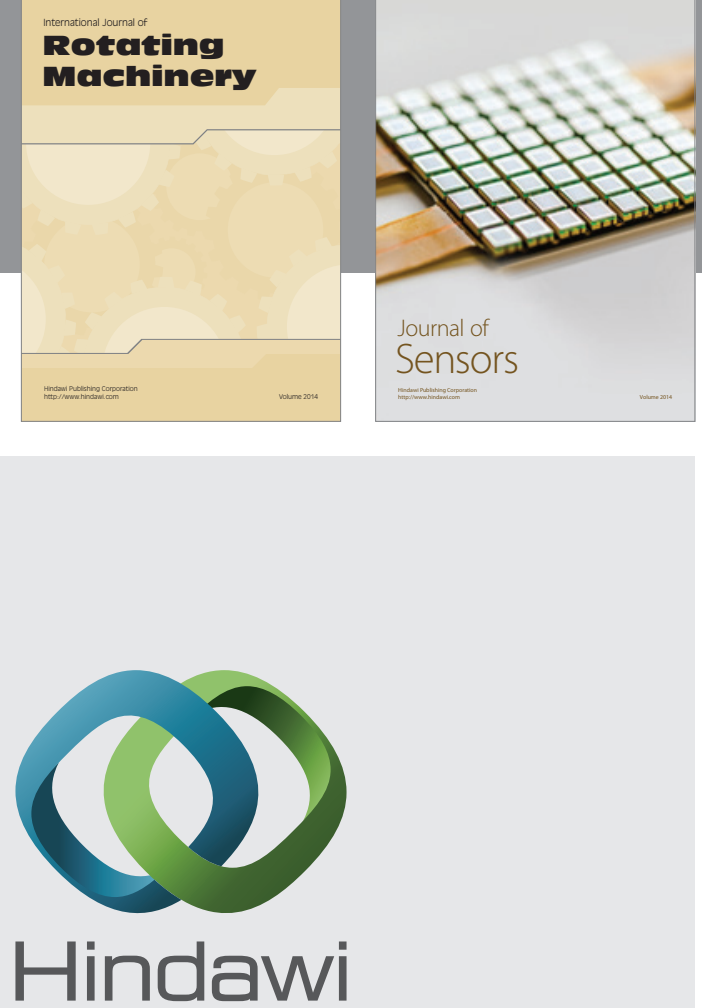

Submit your manuscripts at http://www.hindawi.com
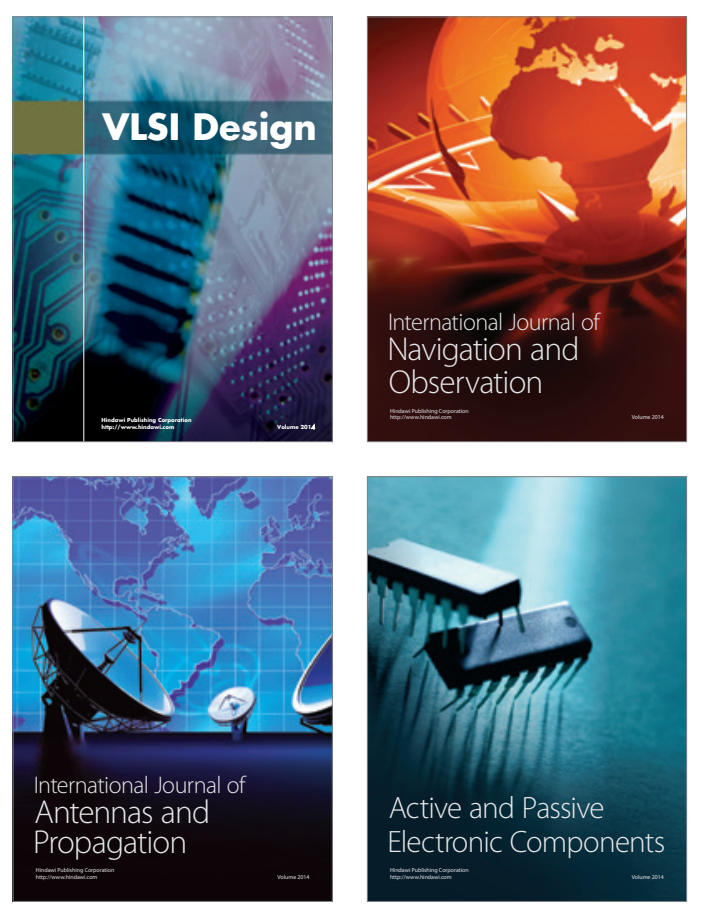
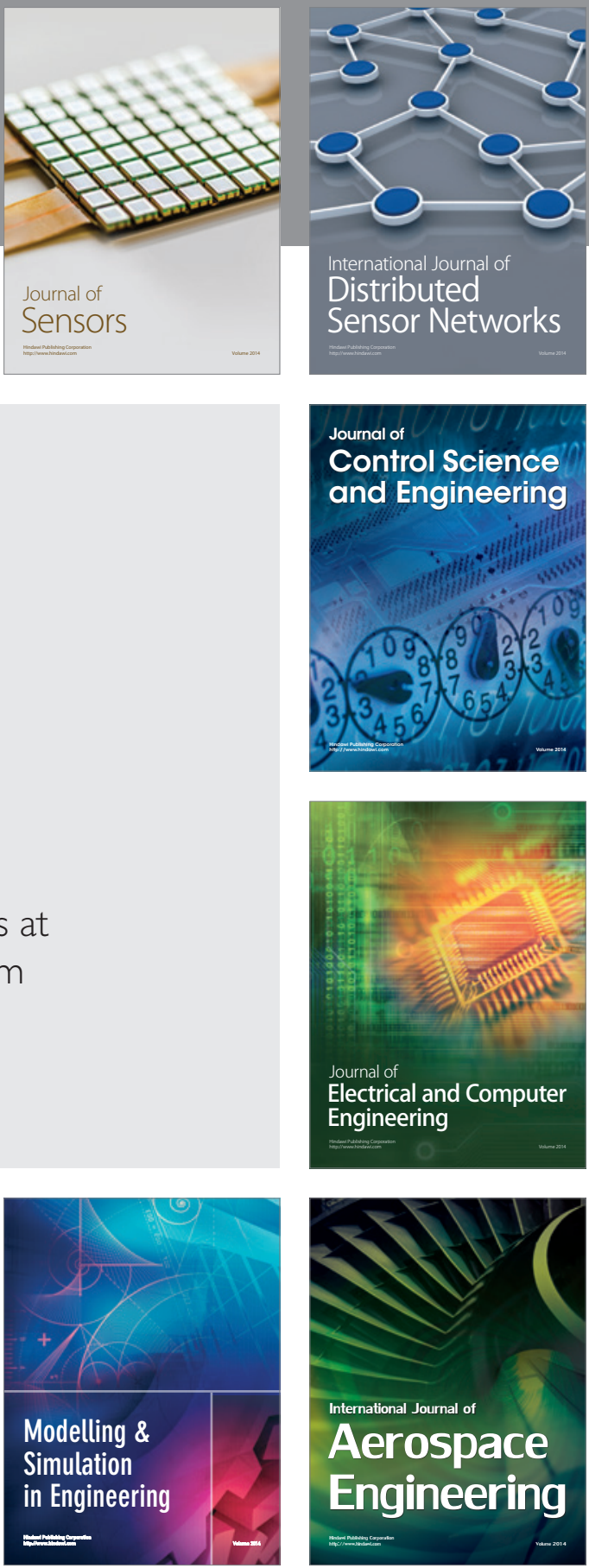

Journal of

Control Science

and Engineering
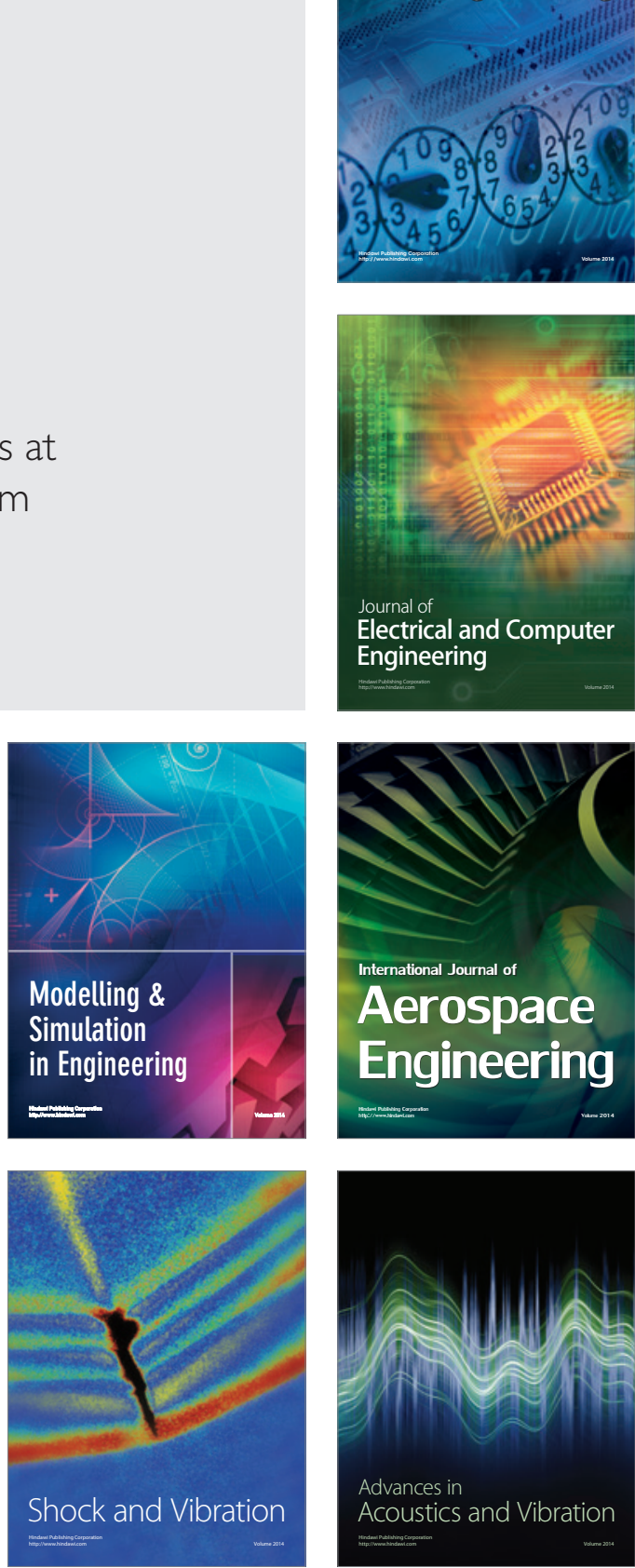